CORPUS $\begin{aligned} & \text { Corpus } \\ & \text { Archivos virtuales de la alteridad americana }\end{aligned}$

Vol. 7, No 1 | 2017

Enero / Junio 2017

\title{
Aportes mapuce para pensar el género
}

Mapuce contributions to think the gender

\section{María Cristina Valdez}

\section{OpenEdition}

\section{Journals}

Electronic version

URL: http://journals.openedition.org/corpusarchivos/1825

DOI: 10.4000/corpusarchivos. 1825

ISSN: 1853-8037

\section{Publisher}

Diego Escolar

\section{Electronic reference}

María Cristina Valdez, «Aportes mapuce para pensar el género », Corpus [En línea], Vol. 7, No 1 | 2017, Publicado el 30 junio 2017, consultado el 22 abril 2019. URL : http://journals.openedition.org/ corpusarchivos/1825; DOI : 10.4000/corpusarchivos.1825

This text was automatically generated on 22 April 2019

Licencia Creative Commons: Atribución-NoComercial 2.5 Argentina (CC BY-NC 2.5 AR) 


\title{
Aportes mapuce para pensar el género
}

\author{
Mapuce contributions to think the gender
}

María Cristina Valdez

\section{Un lugar de enunciación}

1 ¿Es posible reconocerse como indígena feminista? Una problemática que se sigue refiere al orden de los términos que tal como acontece en el caso de reconocerse mujer e indígena no es inocente en el juego de las identidades. Respecto de esto último retomo dos ideas mencionadas por Sousa Santos (1998). La una: "Quien pregunta por su identidad cuestiona las referencias hegemónicas pero, al hacerlo, se coloca en la posición de otro y, simultáneamente, en una situación de carencia y por eso de subordinación" (p.161). La otra: "Es pues, crucial conocer quién cuestiona la identidad, en qué condiciones, contra quién, con qué propósitos y con qué resultados" (p. 162). En lo que sigue, propongo diferenciar entre los procesos de identificación y la asunción estratégica y situada de identidades, la índole política de negociaciones y concesiones y por último la búsqueda y utilización de determinadas teorías en esos procesos.

2 En mi experiencia como mujer, mapuce ${ }^{1} y$ migrante me pregunto: ¿Desde cuándo, para qué y por qué las mujeres mapuce empezamos a hablar del género y a pensarnos en nuestra condición de mujeres? ¿Cómo he llegado a escribir sobre género en el pueblo mapuce? Y ¿cuál ha sido el recorrido reflexivo que me lleva a considerar los estudios de colonialidad y poscolonialidad como los más pertinentes para su abordaje?

"Mi procedimiento es la 'escucha' etnográfica”, sostiene Segato (2013), quien señala cómo las interpelaciones de quienes habitualmente son colocados como objeto de observación y estudio fueron las que la llevaron a desplegar lo que define como una "antropología por demanda"; en otras palabras: "lo que da contenido a mi posición de sujeto investido en la construcción de una marcha descolonial, en este momento, se deriva de las exigencias que me fueron colocadas por demandas, a las cuales he venido respondiendo" (Segato 2013, p 
2). En mi propia trayectoria, las exigencias que me fueron colocadas por demandas vienen de otras mujeres mapuce que apelaron a mi "condición de indígena", no solo para que me esfuerce en una comprensión situada, sino además para que identifique elementos conceptuales y prácticos enraizados en la cosmovisión mapuce, capaces de ofrecer un panorama acerca del género y las relaciones de género.

4 Todo comenzó con una interpelación allá por el año 2003 cuando era estudiante de profesorado, ${ }^{2}$ durante un encuentro de maestros rurales y pueblo mapuce, organizado por el gremio docente de Neuquén y representantes mapuce ${ }^{3}$ en Laguna Blanca, Zapala. Quizás ese fue el primer encuentro en el que participé o el segundo, pero marcó un antes y un después en mi propio proceso de adscripción identitaria. Estábamos en la habitación para pasar la noche y seguir trabajando el día siguiente, y una mujer mapuce de un grupo de seis me dijo que ellas veían que yo compartía su pensamiento y que debía decidir en "qué lugar quería estar". Un segundo acontecimiento fue el momento en que, asumiéndome mapuce, conversé con estas mismas mujeres sobre mi interés de trabajar el género en el pueblo mapuce. Eso fue dos años después del primer acontecimiento: había avanzado con mi formación y afianzado mi trabajo con estas mujeres. Sin embargo, la respuesta no fue la que yo esperaba, pues estas mismas mujeres me dijeron que el género en el pueblo mapuce no existe como problemática y que "eso de las relaciones de género era algo externo como parte de las influencias que nos atravesaban". En ese entonces sentí cierta incomodidad, pensé en las distancias que me separaban de esas otras con las que me identificaba. Hoy percibo que en esa respuesta no se niega la existencia de relaciones de género desiguales, más bien se enuncia un posicionamiento acerca de cómo las relaciones de género occidentales se impusieron en los pueblos originarios. Este fue mi primer acercamiento a un posicionamiento crítico y decolonial acerca de cómo pensar el género en las sociedades preexistentes a los estados-nación. Y pude advertir la carga etnocéntrica en mi postura al desconocer que el género como categoría no es culturalmente neutral.

5 Lo primero que debía hacer era conocer la cosmovisión mapuce y acercarme a las lógicas en las cuales se inscriben las relaciones de género. Para lograr este cometido queda pendiente aprender el mapuzungun ${ }^{4}$ como requisito para asegurar/me una incorporación pertinente de la cosmovisión a la que me intento acercar no sin interrogantes. Desde mi punto de vista, siguen siendo objeto de interpelación los lugares diferenciales que mujeres y hombres ocupan/ocupamos en diferentes espacios, y las responsabilidades que se asumen en movilizaciones y ceremonias. Estos espacios refieren a dos lugares distintos, uno podríamos denominarlo "un espacio otro o intercultural" y el otro, un espacio "propio y autónomo". Como conjetura estimo que en los espacios interculturales la división de género sigue sosteniéndose en términos dicotómicos. ${ }^{5}$ Sin embargo, es necesario indagar críticamente mi punto de vista para profundizar acerca de qué hay de dicotómico en la complementariedad mapuce y qué de complementario en la lógica dicotómica de Occidente.

6 Esta última idea trae a escena, por un lado, la cuestión de las identidades esencializadas que suelen adjudicarse/nos a los indígenas así como a la forma en que recurrimos a determinados esencialismos para diferenciarnos. Por otro, a los procesos de descolonización que debemos iniciar en la configuración de nuestra forma de entender el mundo. En lo que al género refiere, implica comprender la colonialidad de género y ampliar las reflexiones acerca de las nociones de dualidad, reciprocidad y complementariedad. 
7 Mi lugar de enunciación es la interseccionalidad ${ }^{6}$ de las múltiples subordinaciones sin que una $u$ otra se priorice y pueda pensarse por separado de las otras. Resulta oportuno atender el modo en que estas ubicaciones interpelan la lógica de clasificación del proyecto de la modernidad. En efecto, la ubicación "mapuce", o cualquier otra que estratégicamente asumamos, en principio, no excluye, ni va necesariamente atada, a una o unas determinada/s ubicaciones de clase, de edad, de géneros, sexualidades, etc. Siguiendo a Lugones (2008) se plantea la necesidad epistemológica, teórica y política de considerar la interseccionalidad de todas esas ubicaciones construyendo un entramado entre estas categorías que conforman lo que denomina "sistema moderno colonial de género", para poder así visibilizar lo que queda oculto cuando dichas categorías se conceptualizan separadamente.

\section{Una concepción mapuce del género como insurgencia epistémica}

8 Problematizar mi condición de género fue posible en el camino compartido con otras mujeres; he llegado a la conclusión de que es indispensable la construcción de una concepción mapuce del género, que reivindique la cosmovisión indígena como fundamento y retome los aportes de discursos provenientes de las epistemologías de la disensión.

Una perspectiva de género "otra" que toma como punto de partida el mapuce kimvn," distanciándose de las concepciones arcaizantes, esencialistas y folklóricas y entendiéndolo como un cuerpo de saberes en construcción. Que reconoce el "epistemicidio", es decir, el silenciamiento y la destrucción sistemática de las condiciones de producción, circulación y ampliación del conocimiento propio. El "epistemicidio" incluyó prácticas que van desde la prohibición de hablar el mapuzugun hasta el exterminio focalizado de los/las maci/autoridad filosófica a partir de la incorporación del territorio mapuce a los estados nacionales argentino y chileno, a fines del siglo XIX, bajo matrices coloniales de poder y saber. ${ }^{8}$

10 La emergencia del mapuce kimvn solo puede tener lugar en tanto "insurgencia epistémica", que pone en cuestión estas matrices. Generar un concepto enraizado en la cosmovisión comprende hacerlo desde el mapuzugun, en donde la base epistemológica está articulada a un idioma que está situado desde un territorio que sostiene al concepto en su relación con todos los demás elementos. La emergencia del mapuce kimvn requiere diferenciar entre el salvataje de conocimientos locales, los procesos que llevan a poner en tensión el estatuto mismo del saber y los sentidos y estrategias involucrados en la validación y proyección de estos conocimientos.

11 Esta construcción se propone en un contexto en el que circula la idea de concentrar las estrategias de lucha en lo territorial y político, donde el abrir el tema de la colonialidad de género aparece como "distracción". Hay que considerar que las interpelaciones, en cuanto a las "relaciones de género", muchas veces provienen del "afuera" y no en pocas ocasiones, desde perspectivas que toman lo occidental como universal. No es solo en los pueblos originarios/indígenas que la colonialidad de género constituye a la colonialidad del poder. Es necesario relacionar pero no confundir esta constitución del género y del poder entre ambas sociedades. ${ }^{9}$ Las políticas estatales de desintegración comunitaria provocan diferencias específicas habida cuenta de la ancestralidad de relaciones duales, 
recíprocas en la cosmovisión y en la sociedad mapuce con sus contradicciones e historicidad.

12 Un contexto en el que el pueblo mapuce construye saberes desde el rescate, la vigencia y la proyección biocultural con identidad. La decolonización del saber no puede realizarse dentro de los marcos excluyentes del pensamiento occidental, sino que por el contrario, necesita fundarse o refundarse desde la emergencia de saberes 'otros'. La discusión es entonces epistemológica y quienes la están llevando a cabo son las mujeres indígenas, en este lado las mujeres mapuce, que en sus luchas cotidianas proponen volver a educación autónoma y proyectar el conocimiento propio.

Sostener una perspectiva mapuce de género supone observar la existencia del género en la sociedad mapuce antes de la colonización. ¿Qué consecuencias epistemológicas, políticas tiene el considerar que existía el género en la sociedad mapuce precolonial y que es con la intervención colonial que las jerarquías que formaban parte del orden comunitario preinstrucción experimentan una intensificación? Existen conceptos mapuce que permitirían caracterizar el género, tales como: dualidad, complementariedad y reciprocidad. Estos caracterizan las relaciones de género pero no le son específicos, dado que sirven para hablar de las relaciones en general en las que están incluidas las personas o ce y los demás newen (fuerza) que conforman el waj mapu (territorio). Es pertinente matizar el contenido de estas nociones y preguntarnos ¿siempre ha sido así? Asumimos que no, el trabajo decolonial conlleva una búsqueda que no estaría en una escena primigenia y esencial, sino en una historicidad en la que esas mismas categorías cobran sentido.

En los intercambios con otras mujeres no mapuce se nos cuestiona el acatamiento a cierto orden de jerarquía que organiza la vida comunitaria. Este cuestionamiento se articula a la aseveración anterior, lo que no significa desconocer la existencia de jerarquías de diferenciación entre lo femenino y lo masculino previas al orden colonial moderno. Pero entonces ¿qué fue lo que pasó con estas jerarquías en el pasaje de un orden a otro? Siguiendo a Segato (2013), se puede ponderar lo acontecido como un agravamiento e intensificación en el que estas jerarquías son reinterpretadas en un orden superjerárquico. Lo que se registra es la ruptura y continuidad del orden a la luz del nuevo orden moderno y su discurso igualitario convirtiendo un orden jerárquico preintrusión a partir de influencias cruzadas entre estos órdenes. Sin embargo, las condiciones de posibilidad para ejercer efectivamente esas influencias estuvieron dadas por las relaciones de poder diferencial en una y otra sociedad. Esta situación puede explicar, en parte, por qué las influencias benéficas aportadas por "la aldea" no lograron volverse legítimas y por qué su continuidad estuvo asegurada por la memoria histórica traducida en actos de quienes nos consideramos parte o herederos de ese otro mundo. La distancia y exterioridad propias del ejercicio del poder en el mundo de la colonialidad se traducen al mundo de "la aldea". "La captación del género pre-intrusión por el género moderno [implica también] el secuestro de toda política” (Segato 2013, p. 87).

Las mujeres mapuce ocupan mayoritariamente lugares vinculados al ámbito educativo, son "reconocidas" como responsables de la posesión, vigencia y proyección del mapuce kimvn. Estos lugares, en el marco de un movimiento que se define principalmente político, debieran habilitar que más mujeres ocupen cargos ligados a la toma de decisiones y que las demandas epistemológicas sean prioritarias. Pero no es esto lo que acontece; entiendo que revisar las demandas epistemológicas supone examinar críticamente las relaciones de género en las organizaciones indígenas. 
16 No obstante, es a las cuestiones "epistemológicas" a las que se recurre para legitimar la ancestralidad y autonomía de las propuestas. Por ejemplo, en los proyectos vinculados a la defensa de los derechos territoriales, hace años se favorece la presentación de evidencias materiales y simbólicas que den cuenta de la posesión y relación continua de las comunidades con los territorios. En la construcción de estas evidencias y de "otros" mapas que disputen a los de la cartografía oficial participan mayoritariamente mujeres en talleres de la cosmovisión y el renombrar. ${ }^{10}$ En estos talleres, los mapas son construidos comunitariamente apelando a diversas fuentes y al mapuzungun. Son estos espacios habilitados por mujeres mapuce los que tienen el potencial para imaginar formas de habitar decolonial. La construcción de "otros" conocimientos se ancla en la memoria comunitaria atravesada por el género y la generación.

17 Así, creemos que una perspectiva mapuce del género debe ser pensada en este devenir, reconociendo su basamento epistémico en relaciones de otro tipo que habilitan configuraciones identitarias complejas. En este marco, al destacar el carácter dual de la constitución identitaria mapuce podemos considerar en una individualidad rasgos femeninos y masculinos, como producto de un devenir en el que intervienen la familia, la comunidad, nuestro kvpan (origen familiar) y tuwvn (origen territorial). Estos fundamentos distancian el género de las relaciones directas entre anatomía e identidad, entre cuerpo y sexualidad. A la vez, permiten conjeturar que el devenir mujer $u$ hombre no son las únicas opciones disponibles, tampoco son opciones fijas, y que constituirse hombre-mujer - por nombrar las opciones para las que existe un lenguaje- no solo depende de las construcciones socioculturales. Hay "dispositivos" que participan en este devenir que exceden lo que acontece en este mundo, como los pewma (sueños).

La perspectiva mapuce de género se propone recontextualizar la identidad de las mujeres a partir de un nuevo vínculo, distanciándose del feminismo liberal y la polarización individuo-estado. Reivindicar este vínculo requiere pensar en otra trama epistémica en el que la memoria es también un campo de disputa de género. Esta propuesta es un proyecto político en construcción que discute con la pretensión igualitaria entre los géneros del feminismo liberal tensionándola con las nociones de dualidad y complementariedad, celebrando el derecho a la autonomía y a la diferencia.

\section{BIBLIOGRAPHY}

Curiel, O. (2007). Crítica poscolonial desde las prácticas del feminismo antirracista. Revista Nómadas, 26, 92-101.

Lugones, M. (2008). Colonialidad y género. Tabula Rasa, 9, 73-101.

Mahmood, S. (2008). Teoría Feminista y el agente dócil: algunas reflexiones sobre el renacimiento islámico en Egipto. En L. Suárez Navaz y R. Hernández Castillo (Eds.), Descolonizando Feminismo: Teorías y Prácticas desde los Márgenes, (pp. 162-214). España: Cátedra. 
Segato, R. (2013). Género y colonialidad: del patriarcado comunitario de baja intensidad al patriarcado colonial moderno de alta intensidad. En R. Segato (Comp.), La Crítica de la Colonialidad en ocho ensayos. Y una antropología por demanda, (pp. 69-99). Buenos Aires: Prometeo.

Sousa Santos, B. (1998). Modernidad, identidad y cultura de frontera. En B. Sousa Santos, De la mano de Alicia. Lo social y lo político en la posmodernidad, (pp.161-188). Bogotá: Uniandes.

Sousa Santos, B. (2010). Más allá del pensamiento abismal de las líneas globales a una ecología de saberes. En B. Sousa Santos, Descolonizar el saber, reinventar el poder, (pp. 29-60). Montevideo: Trilce.

\section{NOTES}

1. En este escrito se hará uso del mapuzungun, idioma originario del pueblo mapuce, utilizando para su escritura el grafemario Raguileo, el cual ha sido adoptado como propio en ejercicio de la autodeterminación lingüística, dado que respeta la fonética mapuce y rompe con la castellanización de la escritura. Se diferenciarán las palabras en mapuzungun con cursivas.

2. Estudiaba el Profesorado en Ciencias de la Educación en la Universidad Nacional del Comahue. Realizaba investigación sobre educación intercultural en el del Centro de Educación Popular e Intercultural (CEPINT).

3. Las mujeres mapuce eran miembros del Centro de Educación Mapuce Norgvbamtuleayiñ (CEMN), creado en 1996 con mandato de la Confederación Mapuce de Neuquén (CMN). Trabaja en el fortalecimiento de la educación autónoma mapuce y la elaboración de nuevas políticas educativas interculturales.

4. Hacer traducciones literales del mapuzugun al castellano vacía de contenido filosófico y político el pensamiento (rakizuam). Un solo concepto en mapuzugun encierra una narración envolvente. La traducción (práctica por otra parte insoslayable en tanto gran parte del pueblo mapuce en Neuquén no habla mapuzugun) ubica en la encrucijada de tener que explicar (se) y explicar (nos) ideas y sentimientos, corriendo el riesgo de quedar atrapados en los significados con los que el poder lingüístico hegemónico los dice, los representa y los habla.

5. En experiencias de educación intercultural, los referentes mapuce generalmente son hombres, en el entendimiento de que los interlocutores proceden de manera diferencial al tratar con un varón.

6. Este concepto permite referir a la relación entre raza y género del pensamiento dicotómico moderno. La lógica de la opresión necesita compartimentarse y permanecer oculta para ser efectiva.

7. Ver proyectos de investigación C099. Facultad de Ciencias de la Educación. Universidad Nacional del Comahue. (2013-1015).

8. Se trata de una geopolítica del conocimiento cuya hegemonía epistémica surge del singular poder de nombrar por primera vez, crear fronteras, decidir cuáles conocimientos y comportamientos son o no legítimos, y establecer una visión de mundo dominante. Dicha concepción es impuesta a los colonizados para subalternizar sus culturas y sus lenguas.

9. Ver proyecto de investigación C082. Facultad de Ciencias de la Educación. Universidad Nacional del Comahue (2013-1015).

10. . Informe final Relevamiento territorial, histórico, social y cultural de la comunidad mapuce Lof Paicil Antriao: Huellas y senderos. Observatorio de Derechos Humanos y Pueblos indígenas. Neuquén, Argentina. 2013. 


\section{ABSTRACTS}

The paper presents the contributions of a Mapuce perspective on gender to the discussions in this field, as an intervention in the debate to which we have been invited. At first it refers to the situated character of these reflections, traversing events of the experience of the author as a woman, Mapuce and migrant. It recognizes the way subordinate terms actually intersect, as opposed to the compartmentalized logic of modern dichotomous thinking and making apparent the domination pattern that supports it. It then presents the Mapuce conception of gender and its contributions, and shows, on the one hand, how an "other" conception of gender requires different epistemological and worldview frameworks that convey a non-antagonistic becoming conception in which being a man or woman are not the only options available; on the other hand, the way in which the coloniality of the concept of gender affected the precolonial Mapuce society with consequences traceable to the present. Finally, it challenges the liberal feminist equality claim opposing it to the Mapuce notions of gender.

El trabajo expone los aportes que puede realizar una perspectiva mapuce sobre el género a las discusiones en este campo, como intervención en el debate al que se nos ha invitado. Al principio se refiere al carácter situado de estas reflexiones recorriendo acontecimientos de la experiencia de la autora en su condición de mujer, mapuce y migrante. Se reconoce la interseccionalidad de las subordinaciones, oponiéndose a la lógica compartimentada del pensamiento dicotómico moderno y evidenciando la matriz de dominación que la sostiene. Luego se avanza en presentar la concepción mapuce del género y sus aportes. El escrito se detiene en mostrar, por un lado, cómo una concepción "otra" sobre el género requiere fundarse en otros marcos epistemológicos y cosmovisionarios que contienen una concepción del devenir no antagónica, en la que ser hombre o mujer no son las únicas opciones disponibles. Y por otro, la forma en que la colonialidad de género incidió en la sociedad mapuce precolonial con consecuencias observables en la actualidad. Para finalizar se discute con la pretensión igualitaria del feminismo liberal poniéndolo en tensión con las nociones mapuce para pensar el género.

\section{INDEX}

Keywords: Coloniality of gender, intersectionality, epistemic insurgency, Mapuce people Palabras claves: Colonialidad de género, interseccionalidad, insurgencia epistémica, pueblo mapuce

\section{AUTHOR}

\section{MARÍA CRISTINA VALDEZ}

Instituto Patagónico de Estudios en Humanidades y Ciencias Sociales, Consejo Nacional de Investigaciones Científicas y Técnicas (CONICET), Universidad Nacional del Comahue y Lof Puel Pvjv, Argentina 
Correo electrónico: riosmelube@yahoo.com.ar 